\title{
SMOOTHED HEIKIN-ASHI ALGORITHMS OPTIMIZED FOR AUTOMATED TRADING SYSTEMS
}

\author{
Cristian Păuna ${ }^{199}$
}

https://doi.org/10.31410/itema.2018.514

\begin{abstract}
Heikin-Ashi is the Japanese term for "average bar". This methodology is well known as one of the methods to identify and follow the trends using a price time series in financial markets. Nowadays, in the first decades of the 21st century, in the electronic trading environment, with very volatile price market conditions, using the Heikin-Ashi method gets new and special connotations especially when it is about the high-frequency trading. It was found that combining the classical Heikin-Ashi candlesticks with modern limit conditions reliable trading algorithms can be generated in order to produce a good trading return with automated trading systems. This paper will present several trading algorithms based on Heikin-Ashi method for algorithmic trading especially adapted for high-frequency trading systems. It will be revealed how the trading signals can be automatically built and used in order to automate the trading decisions and orders. Exit signals will also be discussed. Trading results obtained with the presented algorithms for Frankfurt Stock Exchange Deutscher Aktienindex Market will be displayed in order to qualify the methods and to compare them with any other trading strategies for high-frequency trading. As conclusions, Heikin-Ashi combined with special limit conditions can generate reliable trading models for algorithmic trading.
\end{abstract}

Keywords: algorithmic trading, automated trading systems, Heikin-Ashi

\section{INTRODUCTION}

$\mathrm{I}^{\mathrm{n}}$ $\mathrm{n}$ the first decades of the 21 st Century "the development of e-businesses represents an important factor in boosting the growth and prosperity of the human society". [1] In the new electronic trading environment of the financial markets, automated trading systems (ATS) have become an important part in any modern financial investment company. "The development of the information and communication technology leads to the creation of new business models and decision support systems”. [2]

"In electronic financial markets, algorithmic trading (AT) refers to the use of computer programs to automate one or more stages of the trading process". [3] The trading decisions and orders are made partially or completely automatically nowadays by computers using advanced mathematical algorithms. "High-frequency trading (HFT) is a type of algorithmic trading characterized by high speeds, high turnover rates, and high order-to-trade ratios that leverages high-frequency financial data and electronic trading tools". [4]

There is a considerable number of trading strategies and models in the literature, but not all of them are adapted for algorithmic trading. Nowadays the algorithmic traders "represent $52 \%$ of market order volume and 64\% of nonmarketable limit order volume". [5]. Different analytical

\footnotetext{
${ }^{199}$ Economic Informatics Doctoral School, Bucharest Academy of Economic Studies, 11th Tache Ionesc Str., Bucharest, Romania

This paper was co-financed by the Bucharest Academy of Economic Studies during the PhD program
} 
trading models that can be automated for the financial markets are presented by Larry Connors in [6] and [7] with proved reliable results. Using the genetic based algorithms, interesting researches are presented by Yong $\mathrm{Hu}$ [8] and José Manuel Berutich [9]. Hybrid trading strategies are also developed in the last time; a hybrid system is presented by Youngmin Kim [10]. A significant number of the trading strategies that can be automated for the stock exchanges are adapted from the currency market; some reliable strategies are presented by Kathy Lien in [11] and [12]. Considering the current academic literature related with the trading strategies adapted for algorithmic trading, the subject about how the Heikin-Ashi transformation can be used to detect the trend and to automate the trading signals is not treated. This is the purpose filled by the current paper.

This paper will present some algorithms optimized especially for HFT. The models presented here are based on the Heikin-Ashi (HA) methodology. HA is the Japanese term (平均足) for the "average bar" and represents a transformation of the price time series into a new series with the purpose to filter the noise and to obtain a graphic representation for the price trend. The HA model is well known as a good method to identify and follow the trend in financial markets. This paper will present how the HA methodology can be combined with modern limit conditions (LC) in order to improve the trading efficiency and to automate the trading decisions for inclusion in ATS.

It will be revealed the way how the trading signals based on HA can be automatically built and used in order to automate the trading activity. Exit signals will also be discussed and built using the same technique in order to automate the exit procedures. To qualify the presented methods and to compare them with any other trading strategies for HFT, trading results will be presented in the last part of the paper. These results were obtained with a HFT of the Frankfurt Stock Exchange Deutscher Aktienindex DAX30 Market [13]. The results will qualify the signals obtained with HA as to be reliable trading signals for AT that can be included and optimized for any ATS.

\section{HEIKEN-ASHI TECHNIQUE}

It is well known that a price time series in financial market is given by the four values Open, High, Low and Close price levels for each time interval. The time interval can vary between one second until one month or even one year. Having the values above, noted here with $\mathrm{Oi}, \mathrm{Hi}$, Li respectively $\mathrm{Ci}$ for each $\mathrm{i}$ interval, the time price series can be easily recorded and used further. For an easy view, the four price levels characteristic for each time periods are drawn as candle sticks presented in Figure 1.

Figure 1: Bullish and bearish price time series candlesticks (source: [14])
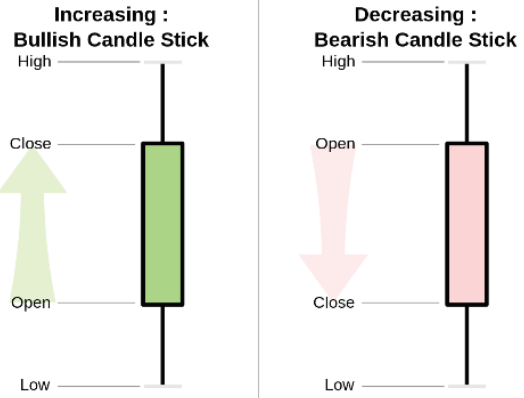

Candlestick charts are thought to have been used and developed "in the 18th century by Munehisa Homma (1724-1803), a Japanese rice trader" [15]. The colors used for the 
candlesticks are at the user disposal. The bullish and bearish differences are usually highlighted using different colors. In the figure 2 it is represented a time price series of the DAX30 index on daily timeframe using the candlesticks representation.

Figure 2: Time price series with candlesticks

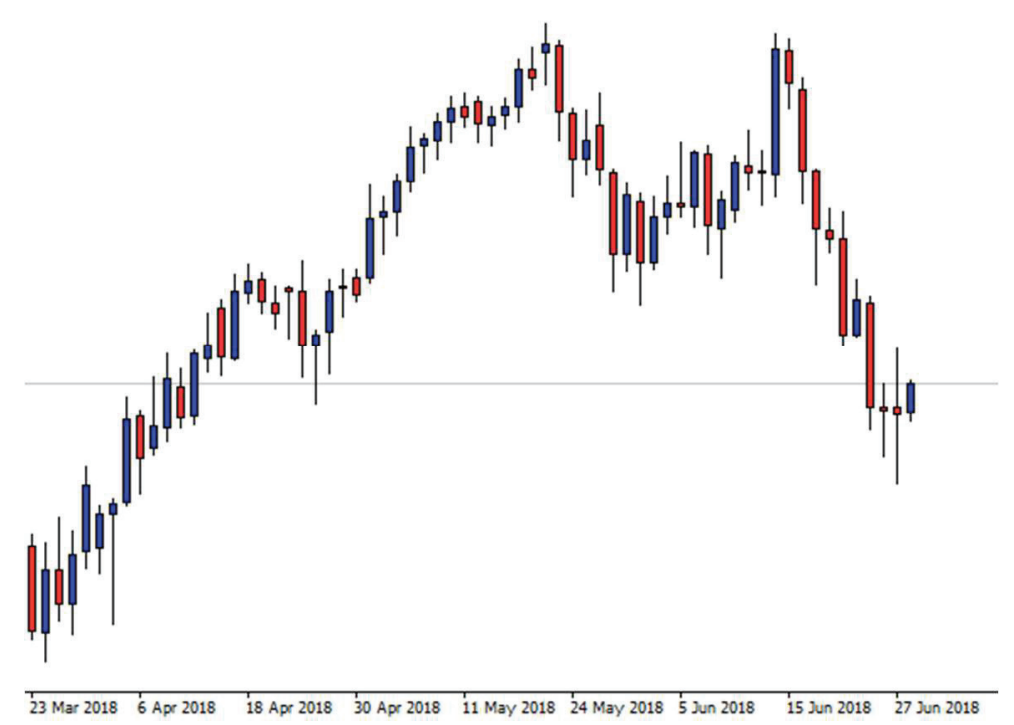

The price trend is not obvious in the figure 2. Even the colors are different for bullish and bearish candles the price tendency is not defined by this graph. The Heikin-Ashi technique will highlight the bullish and bearish tendency of the graph. Reducing the noise and the volatility a more accurate image will be obtained. A new price transformation will be made.

For each i time interval, the open level in the new space will be the average level between open and close price of the last interval, the close level will be the average between the forth price levels of the current interval and the low and high will be given by the minimum respectively maximum of the price levels by:

$$
\left\{\begin{array}{c}
\text { Oha }_{i}=\left(O_{i-1}+C_{i-1}\right) / 2 \\
\text { Lha }_{i}=\min \left(L_{i}, O_{i}, C_{i}\right) \\
\text { Hha } a_{i}=\min \left(H_{i}, O_{i}, C_{i}\right) \\
\text { Cha }_{i}=\left(O_{i}+H_{i}+L_{i}+C_{i}\right) / 4
\end{array}\right.
$$

This transformation will gives us a clearer image for the tendency of the price movement. Using one color for the intervals with Ohai $<$ Chai (usually green or blue) and other color for the cases with Ohai $>$ Chai (usual red) we will obtain a graphs like in figure 3. 
Figure 3: Time price series with Heikin-Ashi candlesticks

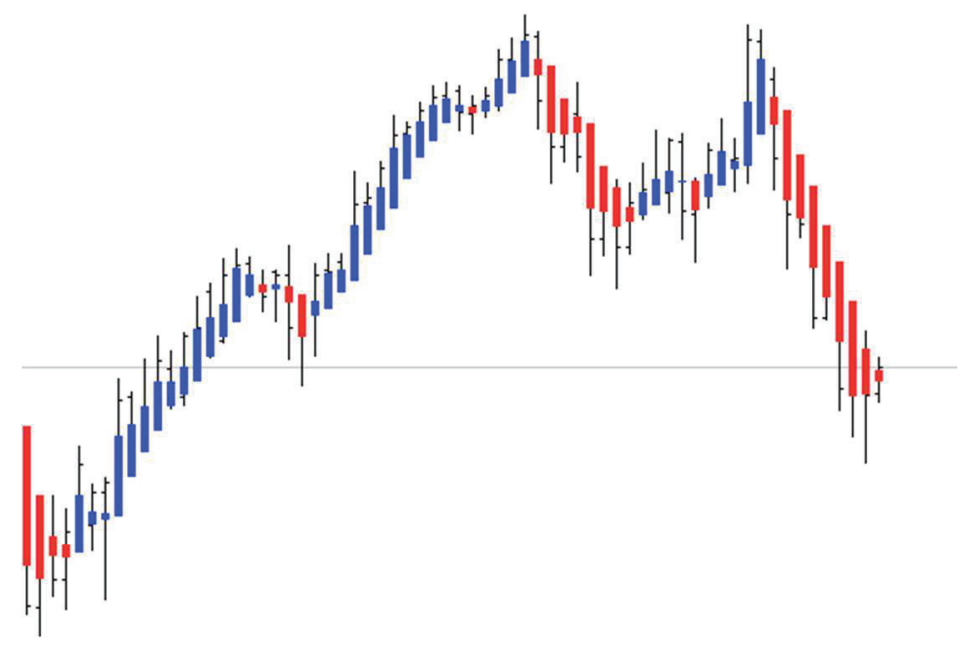

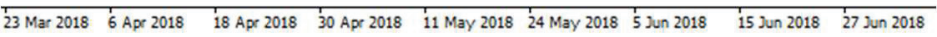

The price time series in the Figure 3 is the same as in the Figure 2. In the figure 3 the transform made by formula (1) is drawn.

\section{SMOOTHED HEIKEN-ASHI}

Even the image is clearer using HA candlesticks, trading in volatile price movements is still difficult. As we can see in figure 3, between the two bullish intervals there are some red HA candlesticks. The graph suggests the long trend is almost finished but a strong continuation contradicts this conclusion after a small interval. To filter the false signals and to give a more stable graph, remaining in strong correlation with the price behavior, a different smoothed HA transform is proposed:

$$
\left\{\begin{array}{c}
\overline{O h a}_{i}=\left(\bar{O}_{i-1}+\bar{C}_{i-1}\right) / 2 \\
\overline{L h a}_{i}=\min \left(\bar{L}_{i}, \bar{O}_{i}, \bar{C}_{i}\right) \\
\overline{H h a}_{i}=\min \left(\bar{H}_{i}, \bar{O}_{i}, \bar{C}_{i}\right) \\
\overline{C h a}_{i}=\left(\bar{O}_{i}+\bar{H}_{i}+\bar{L}_{i}+\bar{C}_{i}\right) / 4
\end{array}\right.
$$

where $\overline{O_{i}}, \overline{H_{i}}, \overline{L_{i}}$ and $\overline{C_{i}}$ represent the averages values for the open, high, low respectively close price levels for a specified $\mathrm{M}$ number of time intervals. Good results are obtained using exponential moving averages [16]. The smoothed HA technique obtained with formulas (2) using exponential averaged of the price levels for $M=6$ time periods is presented in figure 4 . 
Figure 4: Time price series with smoothed Heikin-Ashi technique

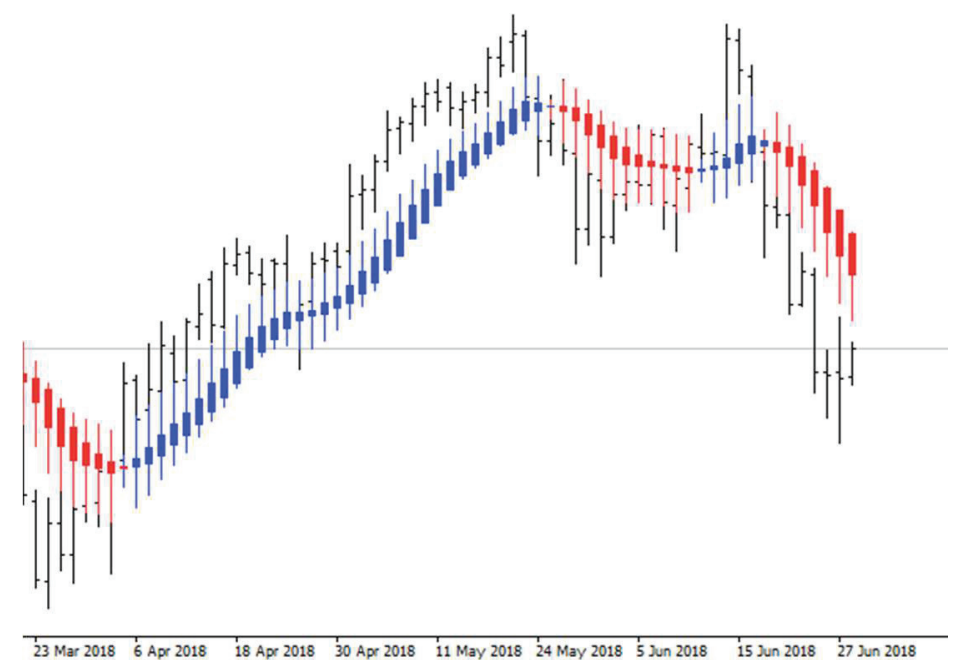

Comparing the figure 3 and figure 4 it can be noticed that the indication given by the candlesticks colors is clearer, a long trade being possible for the entire bullish period included in the figure 4. In practice it is observed that using small period intervals for the moving averages is proper to identify the secondary trends.

For the main trend higher values for the period of the smoothing moving averages must be used. In the figure 5 is presented the same price time series with a modified smoothed HA technique using $M=40$. It can be observed that the price trends are mode obvious. This methodology is more adaptive to catch and follow the large trends. The graph forms the figure 5 was obtained using an exponential moving average for the smoothed HA. The timeframe used in figure 3, figure 4 and figure 5 is the daily timeframe.

Figure 5: Time price series with smoothed Heikin-Ashi technique for longer intervals

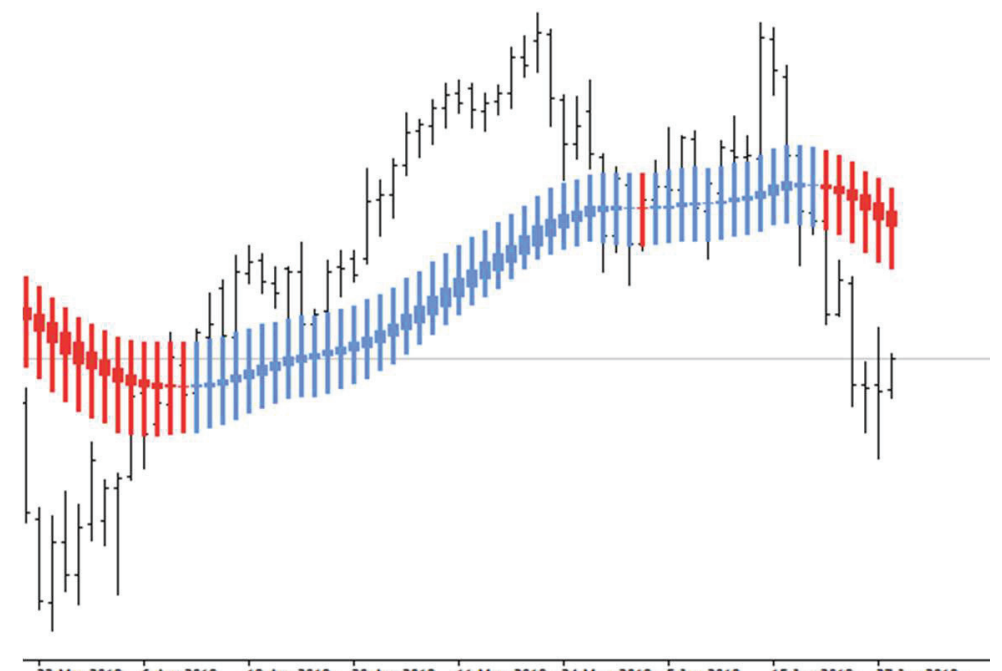


Coding the smoothed HA technique in any multi query programming language is one usual task. To exemplify, in the figure 6 it is presented a sample code for smoothed HA written in Meta Quotes Language [17].

Figure 6: Meta Quotes code for smoothed HA

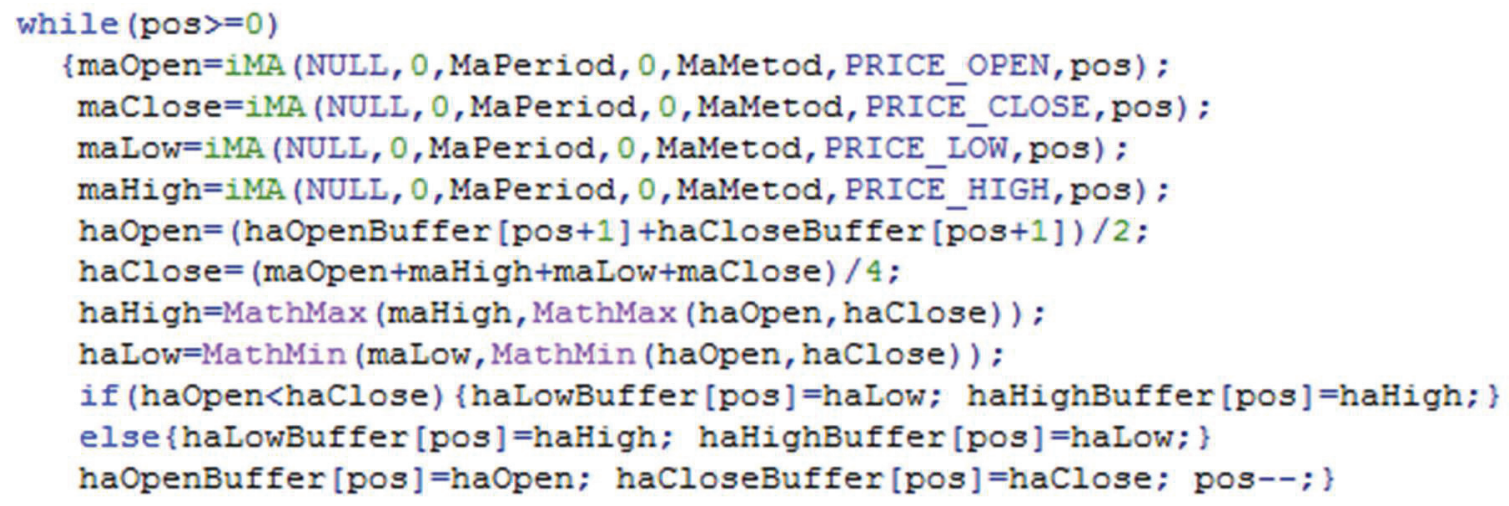

The problem that will be treated in the rest of the paper is how to use smoothed HA price levels in order to build automated trading signals and how to include them into ATS.

\section{TRADING SIGNALS}

Once we have the methodology to obtain HA and smoothed HA candlesticks in order to identify the price trends, in this chapter it will be presented the way to build automated trading signals based on these techniques.

In order to automate the trading decisions and orders, the information regarding the price evolution given by HA series must be transformed into a Boolean variable called trading signal. When the BuySignal variable will have "true" value, a buy trade will be initiated by the ATS. For those markets where sell trades can be considered, when the SellSignal variable will have the "true" value, a short trade will be opened by the automated trading algorithm. For this purpose, the price behavior must be included in these signal variables. The way to build the trading signals based on smoothed HA technique is:

$$
\left\{\begin{array}{l}
\text { BuySignal }_{i}=\left(\overline{\text { Cha }}_{i}>\overline{\text { Oha }}_{i}\right) \wedge\left(\overline{H h a}_{i}>\overline{\text { Lha }}_{i}\right) \\
\text { SellSignal }_{i}=\left(\overline{\text { Cha }}_{i}<\overline{O h a}_{i}\right) \wedge\left(\overline{\text { Lha }}_{i}<\overline{H h a}_{i}\right)
\end{array}\right.
$$

Using smoothed HA technique exit signals can be also considered when the HA changes the color of the candlesticks:

$$
\left\{\begin{array}{l}
\text { BuyExit }_{i}=\text { SellSignal }_{i} \\
\text { SellExit }_{i}=\text { BuySignal }_{i}
\end{array}\right.
$$

The exit signals given by formula (4) can be optimized finding the best value for the M number of time intervals used in the smoothed HA series. The relations (4) are used for AT, especially when trades are kept longer to catch longer trends. 
The formula (4) is not proper for HFT where the profit target is very small. For HFT additional conditions must be imposed in order to avoid opening trades in the overbought and oversold intervals. These conditions will be presented in the next chapter.

In the figure 5 there is a direct relation between the price tendency and the distance between the Open and the Close of the smoothed HA price levels. When the distance between Ohai and Chai is increasing, the price has a strong movement tendency. When the distance between the open and close of the HA smoothed levels is decreasing, the price is slowing down and it is approaching to change its behavior or trend. This considerations will generate the second type of trading signals based on HA technique. A buy signal can be considered when the open of the smoothed HA is lower than the close and the distance between the two levels is increasing. The sell signal can be made in the same idea for those periods with close less than open of the smoothed HA:

$$
\left\{\begin{array}{l}
\text { BuySignal }_{i}=\left(\overline{\text { Cha }}_{i}>\overline{\text { Oha }}_{i}\right) \wedge \\
\left(\overline{\text { Cha }}_{i}-\overline{\text { Oha }}_{i}>\overline{\mathrm{Cha}}_{i-1}-\overline{\mathrm{Oha}}_{i-1}\right) \\
\text { SellSignal }_{i}=\left(\overline{\mathrm{Cha}}_{i}<\overline{\mathrm{Oha}}_{i}\right) \wedge \\
\left(\overline{\mathrm{Oha}}_{i}-\overline{\mathrm{Cha}}_{i}>\overline{\mathrm{Oha}}_{i-1}-\overline{\mathrm{Cha}}_{i-1}\right)
\end{array}\right.
$$

The relations (5) will generate trades only on the intervals when the price makes significant movements in the direction of the trend. All the rest intervals will be ignored or used in order to close the already opened trades. Decreasing the distance between the open and close of the smoothed HA can be also a good exit signal:

$$
\left\{\begin{array}{l}
\text { BuyExit }_{i}=\left(\overline{C h a}_{i}<\overline{O H a}_{i}\right) \vee \\
\left(\overline{\text { Cha }}_{i}-\overline{\text { Oha }}_{i}<\overline{C h a}_{i-1}-\overline{O H a}_{i-1}\right) \\
\text { SellExit }_{i}=\left(\overline{C h a}_{i}>\overline{O H a}_{i}\right) \vee \\
\left(\overline{\text { Oha }}_{i}-\overline{C h a}_{i}<\overline{\text { Oha }}_{i-1}-\overline{C h a}_{i-1}\right)
\end{array}\right.
$$

The signals (3), (4), (5) and (6) can be applied for different timeframes. These signals can be used for both AT and HFT. For HFT the profit target is small. A significant larger number of trades will be made for HFT. For this case additional conditions must to be imposed in order to avoid opening trades on overbought and oversold intervals.

\section{LIMIT CONDITIONS}

Good results were obtained combining the trading signals presented in the last chapter with limit conditions imposed in the "Price Cyclicality function" [18] values. Noting with PCYi the value of the price cyclicality function, the limit conditions for the long signals can be automated using the next formulas:

$$
\begin{aligned}
& \text { BuySignal }_{i}=\left(\overline{C h a}_{i}>\overline{\mathrm{Oha}}_{i}\right) \wedge \\
& \left(\overline{\mathrm{Cha}}_{i}-\overline{\mathrm{Oha}}_{i}>\overline{\mathrm{Cha}}_{i-1}-\overline{\mathrm{Oha}}_{i-1}\right) \wedge \\
& \left(P C Y_{i}>\rho\right) \wedge\left(P C Y_{i}<\xi\right)
\end{aligned}
$$


where $\rho$ and $\xi$ are two functional parameters that will be optimized for each traded market. The computational way to build the PCY function can be found in the author paper [10]. The parameter $\rho$ assure that the trading signal is not initiated too earlier while the parameter $\xi$ will filter the trades in order not to be opened in overbought intervals. The sell signals using the limit conditions with the PCY function can be built similarly.

It was found that the trading signals (7) can open trades even the price trend is not strong enough. In order to filter these cases, a limit condition can be imposed for the gradient of the PCY function. Especially for HFT this filter will ensure good and positive profit expectancy. New trades will be opened by (7) only if the gradient of the PCY function will be higher a specified $\delta$ value:

$$
\begin{aligned}
& \text { BuySignal }_{i}=\left(\overline{C h a}_{i}>\overline{O h a}_{i}\right) \wedge \\
& \left(\overline{C h a}_{i}-\overline{O h a}_{i}>\overline{C h a}_{i-1}-\overline{O h a}_{i-1}\right) \wedge \\
& \left(P C Y_{i}>\rho\right) \wedge\left(P C Y_{i}<\xi\right) \wedge \\
& \left(P C Y_{i}-P C Y_{i-1}>\delta\right)
\end{aligned}
$$

The measure of the functional parameters $\rho, \xi$ and $\delta$ will be found by an optimization process using the time price series for a historical interval. Using an iterative process, the optimal values for each functional parameter will be found checking the trading signals for each time interval in the historical price series. Trading results obtained with (8) will be presented in the next chapter.

\section{TRADING RESULTS}

In this section we will present trading results obtained with the signals detailed above. These results were obtained using TheDaxTrader [19], an automated trading system that uses smoothed HA trading signals in order to generate buy trades for DAX30.

The results presented in table 1 were obtained for HFT of contract for difference (CFD) of DAX30 the period 01.06.2015 - 30.06.2018 with a spread of 1 point using a fixed target of 10 points for each trade. The risk and exposed capital management were made using the "Global Stop Loss Method" presented in the author paper [20].

The smoothed HA trading signals (8) were built for a four hours timeframe interval. An additional condition was imposed regarding the hourly intervals of the executed trades between 8:00 and 16:00 coordinated universal time (UTC) in order to ensure the liquidity on the market.

Table 1. Trading results obtained with smoothed HA trading signals

\begin{tabular}{|l|c|}
\hline Trading signals (8) & results \\
\hline Start trading & 01.06 .2015 \\
\hline Stop trading & 30.06 .2018 \\
\hline CFD spread & 1 \\
\hline Profit target & 10 \\
\hline Number of trades & 78 \\
\hline Total profit & 23,257 \\
\hline Maximal drawdown & 5,442 \\
\hline
\end{tabular}




\begin{tabular}{|l|c|}
\hline Absolute drawdown & 2,204 \\
\hline Maximal RRR & $1: 4.27$ \\
\hline Absolute RRR & $1: 10.55$ \\
\hline
\end{tabular}

The results presented were obtained with the next functional parameters included in the formula (8): $M=40, \rho=5, \xi=95$ and $\delta=8$. The capital evolution due to the smoothed HA signals is presented in figure 7 . In the case presented no losing trades were recorded.

Figure 7: Capital evolution due to HA trading signals

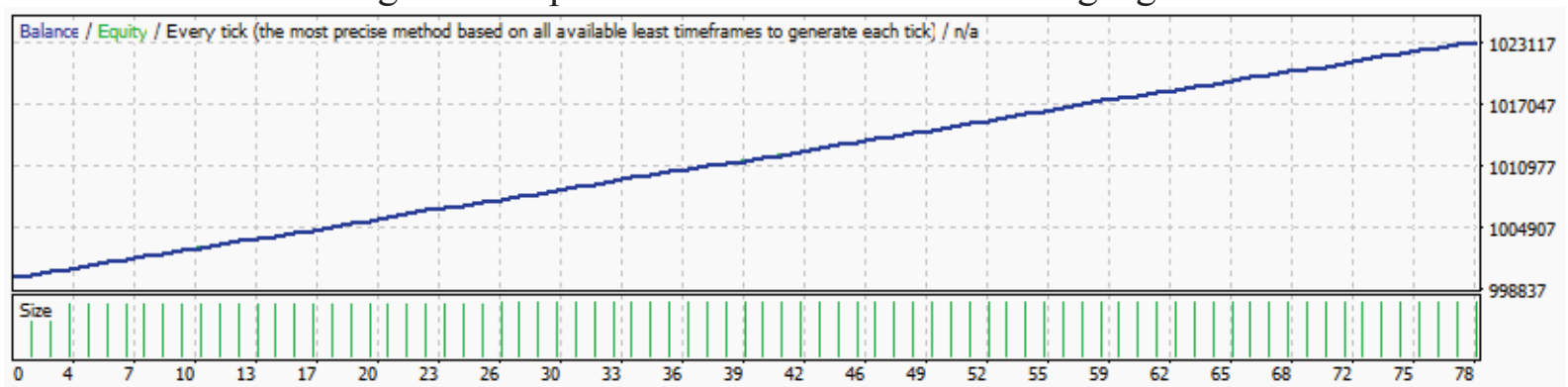

The trading results above were obtained considering only one trade opened in a moment of time. If a trade is opened and a new trading signal appears, no additional trade will be considered until the initial trade is closed. The maximal time interval of the longest trade was 111 hours. In order to reveal the advantages of the Smoothed Heikin-ashi methodology we present a comparison with other known trading methods. The results presented in table 2 were obtained using algorithmic trading in the same period of time $(01.06 .2015-30.06 .2018)$, on the same financial market (DAX30) with the same ten points target.

Table 2. Comparison between trigonometric interpolation and other known trading methodologies

\begin{tabular}{|c|c|c|c|c|}
\hline Trading signals & $\begin{array}{c}\text { Number } \\
\text { of trades }\end{array}$ & Profit & Drawdown & $\begin{array}{c}\text { Risk to } \\
\text { reward ratio }\end{array}$ \\
\hline Smoothed Heikin-Ashi signals & 78 & 23.257 & 5,442 & $1: 4.27$ \\
\hline Moving averages perfect order signals & 96 & 18,045 & 8,712 & $1: 2.07$ \\
\hline Parabolic stop and reverse signals & 102 & 18,816 & 6,011 & $1: 3,47$ \\
\hline Relative Strength Index signals & 68 & 16,372 & 5,901 & $1: 2.77$ \\
\hline
\end{tabular}

The known trading methods used were the "Moving averages perfect order methodology" [11], "Parabolic stop and reverse methodology" [21] and "Relative strength index methodology". [6] Each method was optimized to obtain the best trading efficiency for the financial market used. In table 2 it can be seen that the SHA methodology makes a significant larger number of trades with a very good value for the risk and reward ratio (RRR). These results are an additional confirmation that the method is a reliable trading methodology for algorithmic trading.

The author uses the HSA trading signals presented in this paper since 2013 year. This methodology was included in TheDaxTrader automated trading software. With a proper optimization parameters set, the HSA trading signals generate only profitable trades in the stock markets. This methodology was tested, implemented and used with the same good results for a representative number of financial markets: Deutscher Aktienindex (DAX30), Dow Jones Industrial Average (DJIA30), Financial Times London Stock Exchange (FTSE100), Cotation Assistée en Continue Paris (CAC40), Swiss Stock Exchange Market Index (SMI20), Australian 
Securities Exchange Sydney Index (ASX200), Tokyo stock Exchange Nikkei Index (Nikkei225), NASDAQ100 Index, Standard \& Poor's Index (S\&P500) and Small Capitalization US Index (Russell2000). Also with good and stable results the trigonometric interpolation methodology presented in this paper was applied for Gold and Bent Crude Oil financial markets starting with 2015 year.

\section{CONCLUSIONS}

The Heikin-Ashi trading technique can be improved using smoothed methods with different types of moving averages. The smoothed Heikin-Ashi model can be adapted for algorithmic trading and high-frequency trading in the electronic trading today's market conditions. The presented model permits to identify the up and down trends for different timeframes in any financial market. When an uptrend is detected, a buy signal will be generated by the trading software. Similarly, for those markets with profitable sell conditions, when a down trend is detected, sell trades can be considered.

Automated trading signals can be built using the smoothed Heikin-Ashi time series. Both buy and sell trading signals can be automated together with the exit signals using Boolean variables. In order to avoid opening trades in the overbought and oversold price intervals, additional limit conditions must to be imposed for the Smoothed Heikin-Ashi trading model.

Using the price cyclicality model these conditions can be included in the trading signals variables in order to automate the trade decisions. In order to avoid opening trade in the intervals when the price is not making significant movements additional limit conditions can be imposed with the gradient of the price cyclicality function in order to consider only the strong trends. With all these conditions, the smoothed Heikin-Ashi trading signals can produce a significant number of trades with positive profit expectancy.

The presented trading methodology can be optimized based on the functional parameters in order to be traded on different timeframe intervals and different markets. The functional parameters included in the trading signals formulas will be optimized using repetitive algorithms in order to maximize the profit and to reduce the exposure and capital risk. The proper parameter set will be obtained for each traded market.

The presented trading signals can be named reliable, once a period of 36 months produced trading results with 1:4.27 RRR and 1:10.55 absolute RRR. The optimization process of the functional parameters for the trading signals presented can be made using a time price series of the historical price for any market with good liquidity. These signals can be also optimized for algorithmic trading with longer profit target for automated trading or automated investment systems.

Looking at the compared results included in table 2 we can say that the signals obtained using the smoothed Heikin-Ashi methodology are reliable trading signals. The values for the RRR and the number of trades and profit obtained with the presented methodology are a good confirmation for this assumption.

All trading and the exit conditions included in the presented methodology are based on the Heikin-Ashi price transformation and can constitute a stand-alone trading model for automated trading systems. Being exclusively a mathematical model, the methodology presented in this 
paper can be applied with good results for algorithmic trading and high-frequency trading. This model can also be used for manual trading.

\section{REFERENCES}

[1] Dănăiață, D., Margea, C., Hurbean, L., Artene, A.S. (2014) Electronic services for business environment, Procedia - Social and Behavioral Sciences 124, Published by Elsevier Ltd., pp. 351-360, ISSN: 1877-0428, doi: 10.1016/j.sbspro. 2014.02.496

[2] Ghilic-Micu, B., Mircea, M., Stoica, M. (2010) The Audit of Business Intelligence Solutions, Informatica Economică vol. 14, no. 1/2010, pp. 66-77, ISSN 1453-1305

[3] Nuti, G., Mirghaemi, M., Traleaven, P., Yingsaeree, C. (2011) Algorithmic trading, IEEE Journal, Volume 44, Issue 11, pp. 22

[4] Aldridge, I. (2013) High-Frequency Trading: A Practical Guide to Algorithmic Strategies and Trading Systems, 2nd edition, Wiley, ISBN 1-118-34350-6

[5] Hendershott, T., Riordan, R. (2013) Algorithmic Trading and the Market for Liquidity, Journal of Financial and Quantitative Analysis, 48(4), pp. 1001-1024. DOI:10.1017/S0022109013000471

[6] Connors, L., Alvarez, C. (2009) Short Term Trading Strategies That Work, A Quantitative Guide to Trading Stocks and ETFs, Trading Markets Publishing Group, New Jersey, US, ISBN: 978-0-0919239-0-1

[7] Connors L., Alvarez, C. (2009) High Probability ETF Trading 7 Professional Strategies to Improve Your ETF Trading, Connors Research. ISBN: 978-0-615-29741-5

[8] Yong, H., Kang, L., Xiangzhou, Z., Lijun, S., Ngai, E.W.T., Mei, L. (2015) Application of evolutionary computation for rule discovery in stock algorithmic trading: A literature review, Applied Soft Computing, Applied Soft Computing, 36, 534-551. DOI: https://doi.org/10.1016/j.asoc.2015.07.008

[9] Berutich, J.M., Francisco, L., Francisco, 1., Quintana, D. (2016) Robust technical trading strategies using GP for algorithmic portfolio selection, Expert Systems with Applications, Volume 46 Issue C, March 2016 pp. 307-315, Pergamon Press, Inc. Tarrytown, NY, USA. DOI: 10.1016/j.eswa.2015.10.040

[10] Youngmin, K., Wonbin, A., Kyong J.O., Enke, D. (2017) An intelligent hybrid trading system for discovering trading rules for the futures market using rough sets and genetic algorithms, Applied Soft Computing Archive, Volume 55 Issue C, June 2017, pp. 127-140. Elsevier Science Publishers B. V. Amsterdam, The Netherlands, DOI: 10.1016/j.asoc.2017.02.006

[11]Lien. K. (2009) Day Trading \& Swing Trading the Currency Market. Technical and Fundamental Strategies to Profit from market Moves, John Wiley \& Sons. ISBN: 978-0470-37736-9

[12]Lien, K. (2011) The Little Book of Currency Trading. How to make big Profits in the World of Forex, John Wiley \& Sons. ISBN:978-0-470-77035-1

[13]Börse, Frankfurt. (2018) Frankfurt Stock Exchange Deutsche Aktienindex DAX30 Components. Retrieved from http://www. boerse-frankfurt.de/index/dax

[14]Wikipedia encyclopedia. (2018) Candle sticks chart presentation. Available at: https://en. wikipedia.org/wiki/Candlestick_chart

[15]Morris, G.L. (2006) Candlestick Charting Explained: Timeless Techniques for Trading Stocks and Futures, McGraw-Hill, ISBN 0-07-146154-X

[16]Cox, D.R.Sir. (1961) Prediction by Exponentially Weighted moving Averages and Related Methods, Journal of the royal Statistical Society, Series B, Vol. 23, No. 2, pp. 414-422

[17] Meta Quotes. (2018) Online presentation Meta Quotes Language 4. Available at: https://www. metatrader4.com/en/automated-trading/mq14-programming. 
[18]Păuna, C., Lungu, I. (2018) Price Cyclicality Model for Financial Market. Reliable Limit Conditions for Algorithmic Trading, Journal of Studies and Researches of Economic Calculation and Economic Cybernetics, 4/2018, ISSN: 0585-7511

[19]Păuna, C. (2010) TheDaxTrader. Automated trading system. Online software presentation. Available at: https://pauna.biz/thedaxtrader

[20]Păuna, C. (2018) Capital and Risk Management for Automated Trading Systems, Proceedings of the 17th International Conference on Informatics in Economy, pp 183-188. Available at: https://pauna.biz/Capital_and_Risk_Management

[21] Wilder, W. Jr. (1978). New Concepts in Technical Trading Systems. Greensboro, NC: Trend Research. ISBN 978-0894590276 